\title{
Real-world safety of palbociclib in breast cancer patients in the United States: a new user cohort study
}

Daniel C. Beachler ${ }^{1 *}$ (D, Cynthia de Luise ${ }^{2}$, Aziza Jamal-Allial ${ }^{1}$, Ruihua Yin ${ }^{3}$, Devon H. Taylor ${ }^{1}$, Ayako Suzuki ${ }^{4}$, James H. Lewis ${ }^{5}$, James W. Freston ${ }^{6}$ and Stephan Lanes ${ }^{1}$

\begin{abstract}
Background: There is limited real-world safety information on palbociclib for treatment of advanced stage HR+/ HER2- breast cancer.

Methods: We conducted a cohort study of breast cancer patients initiating palbociclib and fulvestrant from February 2015 to September 2017 using the HealthCore Integrated Research Database (HIRD), a longitudinal claims database of commercial health plan members in the United States.

The historical comparator cohort comprised patients initiating fulvestrant monotherapy from January 2011 to January 2015. Propensity score matching and Cox regression were used to estimate hazard ratios for various safety events. For acute liver injury (ALI), additional analyses and medical record validation were conducted.

Results: There were 2445 patients who initiated palbociclib including 566 new users of palbociclib-fulvestrant, and 2316 historical new users of fulvestrant monotherapy. Compared to these historical new users of fulvestrant monotherapy, new users of palbociclib-fulvestrant had a greater than 2-fold elevated risk for neutropenia, leukopenia, thrombocytopenia, stomatitis and mucositis, and ALI. Incidence of anemia and QT prolongation were more weakly associated, and incidences of serious infections and pulmonary embolism were similar between groups after propensity score matching. After adjustment for additional ALI risk factors, the elevated risk of ALI in new users of palbociclib-fulvestrant persisted (e.g. primary ALI algorithm hazard ratio $(H R)=3.0,95 \%$ confidence interval $(\mathrm{Cl})=1.1-8.4)$.

Conclusions: This real-world study found increased risks of several adverse events identified in clinical trials, including neutropenia, leukopenia, and thrombocytopenia, but no increased risk of serious infections or pulmonary embolism when comparing new users of palbociclib-fulvestrant to fulvestrant monotherapy. We observed an increased risk of ALI, extending clinical trial findings of significant imbalances in grade $3 / 4$ elevations of alanine aminotransferase (ALT).
\end{abstract}

Keywords: Palbociclib, Safety, Real-world, Epidemiology, Acute liver injury

\footnotetext{
* Correspondence: dbeachler@healthcore.com

${ }^{1}$ HealthCore, Inc., 123 Justison Street, Suite 200, Wilmington, DE 19801, USA

Full list of author information is available at the end of the article
}

(C) The Author(s). 2021 Open Access This article is licensed under a Creative Commons Attribution 4.0 International License, which permits use, sharing, adaptation, distribution and reproduction in any medium or format, as long as you give appropriate credit to the original author(s) and the source, provide a link to the Creative Commons licence, and indicate if changes were made. The images or other third party material in this article are included in the article's Creative Commons. licence, unless indicated otherwise in a credit line to the material. If material is not included in the article's Creative Commons licence and your intended use is not permitted by statutory regulation or exceeds the permitted use, you will need to obtain permission directly from the copyright holder. To view a copy of this licence, visit http://creativecommons.org/licenses/by/4.0/. The Creative Commons Public Domain Dedication waiver (http://creativecommons.org/publicdomain/zero/1.0/) applies to the data made available in this article, unless otherwise stated in a credit line to the data. 


\section{Background}

Palbociclib was the first cyclin-dependent kinase $4 / 6$ (CDK4/6) inhibitor to receive accelerated approval by the United States (US) Food Drug Administration (FDA) in February 2015 to treat post-menopausal women for advanced stage Hormone Receptor Positive (HR+)/Human Epidermal Growth Factor Negative (HER2-) breast cancer in combination with letrozole as initial endocrine therapy [1]. In February 2016, palbociclib in combination with fulvestrant received US approval for the treatment of women with HR+/HER2- advanced or metastatic breast cancer with disease progression following endocrine therapy [1].

Randomized-controlled trials (PALOMA 2/3) [2, 3] demonstrated that palbociclib prolonged progressionfree survival by 5 to 10 months when used in combination with letrozole or fulvestrant compared to endocrine monotherapy. However, adverse events (AEs) and discontinuations due to AEs were more frequent in the palbociclib (with endocrine therapy) arms compared to the placebo-controlled (with endocrine therapy) arms in PALOMA2 (9.7\% vs. $5.9 \%)$ and PALOMA3 (2.6\% vs. $1.7 \%$ ) $[2,3]$. Some of the most commonly occurring (> $10 \%)$ AEs in the palbociclib arms of the trials included neutropenia, infections, leukopenia, and anemia [2, 3]. These trials also revealed an increased risk of grade $3 / 4$ elevations of alanine aminotransferase (ALT), with palbociclib - but were limited in size and breast cancer disease severity in evaluating less common AEs such as acute liver injury (ALI) [1-3]. This study's objectives were to describe the characteristics of new palbociclib users and to evaluate its safety under real world conditions. Specific safety events of interest were evaluated comparing new users of palbociclib-fulvestrant with historical new users of fulvestrant monotherapy. Based on the initial results, further analyses were conducted to further assess ALI and to validate ALI using medical records.

\section{Methods}

\section{Study population and design}

This new user cohort study was conducted using the HealthCore Integrated Research Database (HIRD). The HIRD includes claims for over 50 million commercially insured health plan members from across the US. Patient enrollment data, inpatient and outpatient medical care, and outpatient prescription drug use are tracked longitudinally for each patient.

This study includes descriptive analyses of all palbociclib new users in the HIRD, and comparative analyses of various pre-specified safety events. The incidence of safety events was evaluated in three subgroups of palbociclib users from 01 February 2015 until 30 September 2017: 1) new users of palbociclib-letrozole, 2) new users of palbociclib-fulvestrant, and 3) all other new users of palbociclib (i.e., past fulvestrant/letrozole use or no fulvestrant/letrozole use at time of palbociclib initiation). These palbociclib groups each required individuals to be at least 18 years of age and have at least 3 months of health plan coverage before initiating palbociclib (without prior use of a CDK 4/6 inhibitor (Supplemental Table 2).

For the comparative analyses, we contrasted one of the palbociclib groups, new users of palbociclib-fulvestrant, to new users of fulvestrant monotherapy, because these regimens have similar indications (disease progression following endocrine therapy) and were expected to have good comparability. In contrast, letrozole monotherapy is approved for treating patients with early stage disease, and thus deemed an unfit comparator for the palbociclib-letrozole group [4]. The comparator group in this study included individuals who were newly dispensed fulvestrant monotherapy from 01 January 2011 until 31 January 2015, before palbociclib was available and met inclusion criteria noted for the palbociclib groups (along with requiring $\geq 3$ months with no dispensing of fulvestrant prior to the index date; Supplemental Table 2).

This historical comparator group was chosen owing to enhance comparability with palbociclib-fulvestrant. After palbociclib became available, the decision to add palbociclib versus initiating fulvestrant monotherapy could be related to differences in patient characteristics potentially related to risk of ALI, such as severity of disease.

\section{Follow-up and exposure classification}

For each treatment group, the start of follow-up (index date) was the day after the date of the first dispensing of palbociclib or, for the comparator group, fulvestrant, with a requirement that they had no palbociclib or fulvestrant dispensings in at least the prior 3 months during the study period. Treatment episodes started on the dispensing date, and continued for the number of days supplied, plus 30 days to account for possible nonconcordance of dispensing date and administration. Consecutive dispensings defined in this manner were concatenated into a single continuous treatment episode. Treatment episodes were discontinued after a 30-day gap period without another dispensing or, for palbociclib, after switching to another CDK4/6 inhibitor. If a patient re-initiated palbociclib or fulvestrant monotherapy without a prior censoring/safety event, their subsequent "treated" person-time after re-initiation (treatment episode(s)) was also included. This affected 13\% of palbociclib-fulvestrant patients and $27 \%$ of historical fulvestrant monotherapy patients. Patients were followed while treated until the earliest of the following dates: end of study period (30 September 2017 for palbociclib, 
or 01 February 2015 for fulvestrant monotherapy), end of continuous health plan enrollment, or at the end of all palbociclib or fulvestrant monotherapy treatment episode(s). Follow-up also ended on the date of any occurrence of a safety event being analyzed (as defined in claims). For analysis of each safety event, follow-up was not truncated due to occurrence of another safety event.

\section{Safety events}

To identify safety events, we used algorithms based on the International Classification of Diseases, Ninth and Tenth Revisions (ICD-9/10) diagnosis codes and procedure codes associated with insurance claims (defined in Supplemental Table 1). Many of the algorithms were designed to reduce the possibility of missing a case. Safety events included: neutropenia, febrile neutropenia, leukopenia, alopecia, vomiting, QT prolongation, fatigue, various forms of infection, diarrhea, interstitial lung disease/pneumonitis, anemia, nausea, thrombocytopenia, pulmonary embolism, venous embolism and thrombosis, embolism and thrombosis of unspecified artery, cataracts and other ocular disorders, stomatitis and mucositis, fever, anorexia, peripheral neuropathy, sudden cardiac death, diabetes mellitus, type 2 diabetes mellitus, hyperglycemia, ALI, elevated ALT, elevated aspartate amino transferase (AST), abnormal alkaline phosphatase (ALP), second primary malignancies, and non-melanoma skin cancer. For certain safety events of interest (neutropenia, febrile neutropenia, leukopenia, and anemia) a second more specific algorithm that added more stringent criteria to reduce false-positive errors was also evaluated.

Unless otherwise noted, individuals with a history of a particular safety event (defined by the same algorithm) on or prior to the index date were excluded from the computation of incidence for that event. Certain commonly re-occurring safety events (e.g. nausea, diarrhea) allowed a history of these events on or prior to the index date. These events are all defined in supplemental Table 3.

\section{Additional analyses for ALI}

After identifying an increased risk of ALI among new users of palbociclib-fulvestrant, we conducted additional activities including: development of multiple case definitions (ALI algorithms), medical record validation of ALI algorithms, further control for potential confounding using ALI risk factors, and the addition of a contemporaneous comparator group.

Validation studies of claims algorithms for ALI have reported low sensitivity or specificity with positive predictive values (PPVs) as low as $25 \%$ [5], so we used multiple algorithms for ALI to assess the robustness of the results with respect to ALI definition. The original ALI algorithm included codes for elevated liver enzymes as well as liver necrosis (Supplemental Table 1). After further review, we used a primary ALI algorithm derived from another safety study [6] and a Mini-Sentinel validation study [5] that restricted to inpatient discharge diagnoses for ALI and acute liver failure (not elevated liver enzymes). Inpatient diagnoses identify more serious events and are considered more reliable than outpatient diagnoses which can include rule-out and presumptive diagnoses. We also developed a more sensitive ALI algorithm that included a broader set of codes in both inpatient and outpatient settings [5, 7], and a more specific ALI algorithm restricting to primary inpatient codes that had a high PPV in the Mini-Sentinel validation study (algorithms defined in Supplemental Table 1) [5].

.Medical record validation of potential ALI cases identified by all four ALI claims algorithms involved review of medical records by at least two independent hepatologists with expertise in drug-induced liver injury. We sought medical records for all potential ALI cases identified by the claims algorithms. Hepatologists who were blinded to study treatment adjudicated potential cases as confirmed ALI or non-ALI based on criteria that required elevation in at least one liver enzyme test (e.g., ALT $>3 x$ ULN), specific timing of the enzyme tests, and absence of chronic liver disease [8, 9]. Potential ALI cases that were reviewed but lacked sufficient information in the medical record for adjudication were classified as provisional cases.

We included a contemporaneous comparator of fulvestrant monotherapy which, although smaller and more prone to channeling (i.e. likely to include less severe patients), would not be affected by possible temporal trends (e.g., ICD-9 to ICD-10 code transition in October 2015) that might affect the historical comparator group [10]. The contemporaneous comparator included new users of fulvestrant monotherapy between 01 February 2015 until 30 September 2017, with follow-up censored on the date of initiation of palbociclib or on the date of another previously described censoring criterion.

\section{Statistical analyses}

Study population characteristics were described using measures of central tendency (mean, standard deviation, median, and interquartile range) for continuous variables and frequencies for categorical variables. To assess comparability between treatment groups, absolute standardized differences were computed for each covariate [11].

The incidence of the safety events was calculated for each event in each treatment group by dividing the number of events by the person-time at-risk accumulated in the treatment group. Hazard ratios (HRs) were calculated using Cox proportional hazard regression models, and 95\% confidence intervals (CIs) for the incidence estimates [12]. Propensity score matching was 
used to control for potential confounders [13]. A logistic regression model for propensity score development included covariates that are important predictors of the outcomes of interest [13]. Variables available for inclusion in the propensity score included demographics, medical history, imaging, breast cancer treatments, healthcare utilization, co-morbidities and non-breast cancer related medication within 6 months of palbociclib or fulvestrant initiation [14].

The distributions of propensity scores for each treatment group were examined, and patients having propensity scores outside the region of overlap between the comparison groups were excluded (trimmed) [15]. We stratified the population by propensity score decile using the distribution of propensity scores in the palbociclibfulvestrant population. Each palbociclib-fulvestrant new user was then matched by propensity score stratum to one new user of fulvestrant monotherapy [15]. Variables incorporated in the propensity score for initial analyses included age, region, Deyo-Charlson Index, number of outpatient visits, number of emergency room visits, secondary malignancy (i.e. metastases) to lymph nodes of head, face, and neck, secondary malignancy to other specified sites, secondary malignancy to respiratory and digestive sites (which includes liver metastases), tamoxifen, everolimus, anastrazole, denosumab or pamidronate, exemestane, chemotherapy, corticosteroids, diagnostic imaging, breast cancer surgery, letrozole, HER2 positive therapy, radiation therapy, CT imaging, mammography, MRI imaging, anticonvulsants, antidepressants, sedatives/hypnotics, secondary malignancy to breast, breast cancer diagnosis code, in situ breast cancer diagnosis, hyperglycemia, and cerebrovascular disease.).

To further evaluate ALI, we created a propensity score that included additional baseline covariates that may be associated with ALI. The ALI propensity score included the previously described variables along with chronic liver disease or alcoholism, chronic or acute disease of gallbladder or pancreas, hepatic, biliary or pancreatic cancer, congestive heart failure, and medications associated with ALI (acetaminophen, allopurinol, amiodarone, amitriptyline, + clavulanic acid, aripiprazole, baclofen, ciprofloxacin, clindamycin, clopidogrel, duloxetine, estrogens, fluoxetine, ketoconazole, lisinopril, losartan, mirtazapine, Nitrofurantoin, NSAIDs, omeprazole, paroxetine, phenothiazine, sertraline, statins, tetracycline, trazodone, and trimethoprim). The development of the ALI propensity scores was the same as described above with the exception that strata were defined by propensity score quartiles to improve ability to identify matching comparators.

In sensitivity analyses, we examined the potential effect of unmeasured confounding by calculating the E-value (the associations between the confounder-exposure and the confounder-outcome needed to attenuate the association of interest to a level indicating no effect $(\mathrm{HR})=$
1.0)) [16]. We also examined the possible impact of outcome misclassification of ALI on effect estimates using hypothetical values of misclassification rates [17].

\section{Results \\ Descriptive analyses}

From February 2015 to September 2017, 2795 individuals received at least one dispensing of palbociclib; 2445 of whom met inclusion criteria which required individuals to be at least 18 years of age, have at least 3 months of health plan coverage, and at least 3 months with no dispensing of palbociclib or CDK 4/6 inhibitor prior to the index date of new palbociclib use (Supplemental Table 2). Among the 2445 eligible new users of palbociclib, there were 566 new users of palbociclib-fulvestrant, 1159 new users of palbociclib-letrozole, and 720 other new users of palbociclib. There were 2316 eligible individuals who received at least one dispensing of fulvestrant during the historical comparator period from January 2011-January 2015 and met inclusion criteria (including $\geq 3$ months with no dispensing of fulvestrant prior to the index date; Supplemental Table 2).

The three palbociclib subgroups were similar at baseline (Table 1). Most palbociclib initiators were between the ages of 45-64 (60.9\%), previously used an aromatase inhibitor (62.5\%), had a secondary malignancy/metastasis prior to the index date (87.4\%), and had advanced stage ER+/HER2- breast cancer (93.5\%) [18].Most patients were female, although 53 males (2.2\%) were dispensed palbociclib. Healthcare utilization in the previous 6 months was common (mean number of outpatient visits $=39.7$ ), but surgery (mastectomy/lumpectomy), chemotherapy, and radiation therapy during the same period were less common (each $<20 \%$ ). The most common non-breast cancer related medications received prior to palbociclib initiation included antidepressants (30.5\%), antihypertensives (26.7\%), and corticosteroids (24.8\%). Common co-morbidities included cerebrovascular disease, pure hypercholesterolemia, pathologic fracture, and osteoporosis (each $\geq 8.0 \%$ ).

The incidence rates of safety events after the initiation of palbociclib among all new users are described in Supplemental Table 3. Safety events common to palbociclib users after initiation included neutropenia, anemia, interstitial lung disease/pneumonitis, and serious infections (each incidence rate $>20$ per 100 person-years). Less common safety events included sudden cardiac death, stomatitis and mucositis, febrile neutropenia, and ALI (each incidence rate $<5$ per 100 person-years).

\section{Comparative analyses}

Descriptive characteristics of the historical comparator group of new users of fulvestrant monotherapy are provided in Table 2 and Supplemental Table 4. Before 
Table 1 Select Characteristics of New Users of Palbociclib Identified in the HIRD

\begin{tabular}{|c|c|c|c|c|c|c|c|c|}
\hline \multirow[t]{2}{*}{ Characteristics $^{\mathrm{a}}$} & \multicolumn{2}{|c|}{$\begin{array}{l}\text { All new users } \\
\text { of palbociclib }\end{array}$} & \multicolumn{2}{|c|}{$\begin{array}{l}\text { New users of } \\
\text { palbociclib- } \\
\text { fulvestrant }\end{array}$} & \multicolumn{2}{|c|}{$\begin{array}{l}\text { New users of } \\
\text { palbociclib- } \\
\text { letrozole }\end{array}$} & \multicolumn{2}{|c|}{$\begin{array}{l}\text { All other new } \\
\text { users of } \\
\text { palbociclib }\end{array}$} \\
\hline & $\begin{array}{l}\mathrm{N} / \\
\text { Mean }\end{array}$ & $\% / S D$ & $\begin{array}{l}\text { N/ } \\
\text { Mean }\end{array}$ & $\% / S D$ & $\begin{array}{l}\text { N/ } \\
\text { Mean }\end{array}$ & $\% / S D$ & $\begin{array}{l}\mathrm{N} / \\
\text { Mean }\end{array}$ & $\% / S D$ \\
\hline Overall & 2445 & $100 \%$ & 566 & $100 \%$ & 1159 & $100 \%$ & 720 & $100 \%$ \\
\hline Total duration of follow-up of cohort (in years) & 1540 & & 324 & & 812 & & 404 & \\
\hline Duration of follow-up (in years) & 0.63 & 0.53 & 0.57 & 0.43 & 0.70 & 0.58 & 0.56 & 0.50 \\
\hline Age at index date (in years) & 59.79 & 11.62 & 59.47 & 11.38 & 59.21 & 11.27 & 60.95 & 12.29 \\
\hline \multicolumn{9}{|l|}{ Sex } \\
\hline Male & 53 & 2.17 & $\leq 10$ & $\mathrm{n} / \mathrm{a}$ & 14 & 1.21 & 30 & 4.17 \\
\hline Female & 2392 & 97.83 & 557 & 98.41 & 1145 & 98.79 & 690 & 95.83 \\
\hline \multicolumn{9}{|l|}{ Calendar year of index date } \\
\hline 2015 & 791 & 32.35 & 99 & 17.49 & 456 & 39.34 & 236 & 32.78 \\
\hline 2016 & 942 & 38.53 & 269 & 47.53 & 406 & 35.03 & 267 & 37.08 \\
\hline 2017 & 712 & 29.12 & 198 & 34.98 & 297 & 25.63 & 217 & 30.14 \\
\hline \multicolumn{9}{|l|}{ Geographic region of residence } \\
\hline Midwest & 400 & 16.36 & 95 & 16.78 & 185 & 15.96 & 120 & 16.67 \\
\hline South & 581 & 23.76 & 159 & 28.09 & 288 & 24.85 & 134 & 18.61 \\
\hline Northeast & 696 & 28.47 & 166 & 29.33 & 340 & 29.34 & 190 & 26.39 \\
\hline West & 768 & 31.41 & 146 & 25.80 & 346 & 29.85 & 276 & 38.33 \\
\hline Secondary malignancy to any site (metastasis) & 2137 & 87.40 & 496 & 87.63 & 1044 & 90.08 & 597 & 82.92 \\
\hline Secondary malignancy to Lymph nodes of head, face, and neck metastasis & 690 & 28.22 & 154 & 27.20 & 356 & 30.72 & 180 & 25.00 \\
\hline $\begin{array}{l}\text { Secondary malignancy to Respiratory and digestive system metastasis (includes } \\
\text { liver metastasis) }\end{array}$ & 1058 & 43.27 & 257 & 45.41 & 490 & 42.28 & 311 & 43.19 \\
\hline Secondary malignancy to Metastasis to other specified sites & 2017 & 82.49 & 463 & 81.80 & 978 & 84.38 & 576 & 80.00 \\
\hline Deyo-Charlson comorbidity index (DCl) & 8.39 & 1.90 & 8.52 & 1.76 & 8.48 & 1.70 & 8.13 & 2.25 \\
\hline Advanced stage ER+/HER2- breast cancer & 2285 & 93.46 & 548 & 96.82 & 1083 & 93.44 & 654 & 90.83 \\
\hline Radiation therapy & 483 & 19.75 & 112 & 19.79 & 233 & 20.10 & 138 & 19.17 \\
\hline Chemotherapy & 472 & 19.30 & 100 & 17.67 & 218 & 18.81 & 154 & 21.39 \\
\hline $\mathrm{CT}$ related imaging & 602 & 24.62 & 139 & 24.56 & 333 & 28.73 & 130 & 18.06 \\
\hline Number of outpatient visits & 39.67 & 25.20 & 38.58 & 23.63 & 39.51 & 23.78 & 40.79 & 28.42 \\
\hline Aromatase inhibitor & 1527 & 62.45 & 326 & 57.60 & 815 & 70.32 & 386 & 53.61 \\
\hline HER2+ therapy & 72 & 2.94 & 15 & 2.65 & 35 & 3.02 & 22 & 3.06 \\
\hline Tamoxifen & 552 & 22.58 & 140 & 24.73 & 270 & 23.30 & 142 & 19.72 \\
\hline Fulvestrant & 621 & 25.40 & 238 & 42.05 & 112 & 9.66 & 271 & 37.64 \\
\hline Denosumab or pamidronate & 836 & 34.19 & 206 & 36.40 & 359 & 30.97 & 271 & 37.64 \\
\hline Everolimus & 150 & 6.13 & 40 & 7.07 & 52 & 4.49 & 58 & 8.06 \\
\hline Antihypertensives & 653 & 26.71 & 173 & 30.57 & 293 & 25.28 & 187 & 25.97 \\
\hline Corticosteroids & 606 & 24.79 & 148 & 26.15 & 308 & 26.57 & 150 & 20.83 \\
\hline Lipid lowering agent & 528 & 21.60 & 132 & 23.32 & 251 & 21.66 & 145 & 20.14 \\
\hline Pathologic fracture & 211 & 8.63 & 46 & 8.13 & 110 & 9.49 & 55 & 7.64 \\
\hline Pure hypercholesterolemia & 215 & 8.79 & 48 & 8.48 & 105 & 9.06 & 62 & 8.61 \\
\hline
\end{tabular}

Abbreviations: HIRD HealthCore Integrated Research Database, $N$ number, SD standard deviation, ER estrogen receptor, HER2 human epidermal growth factor receptor 2, CT computed tomography

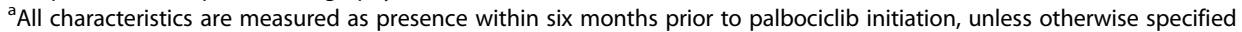




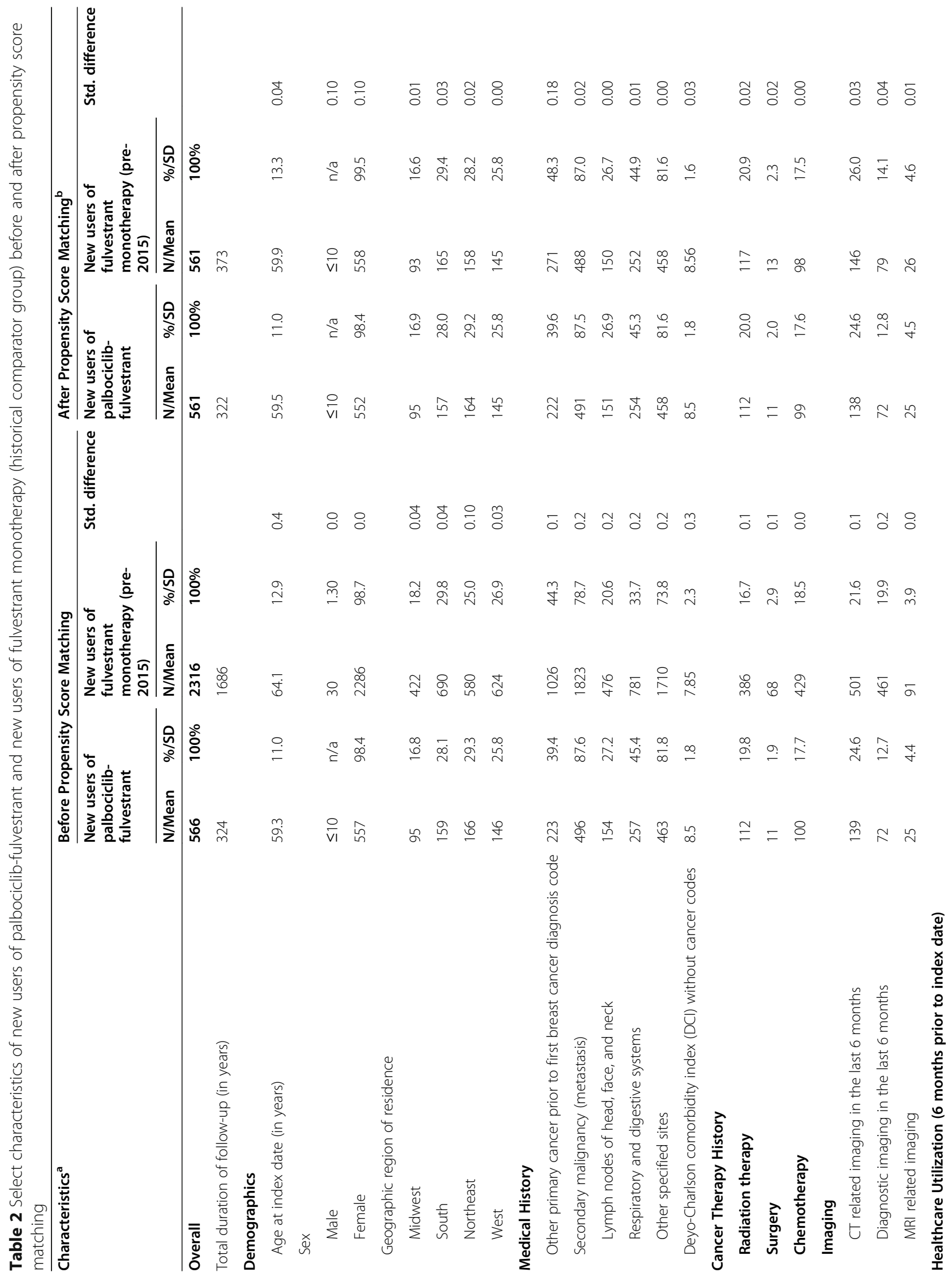




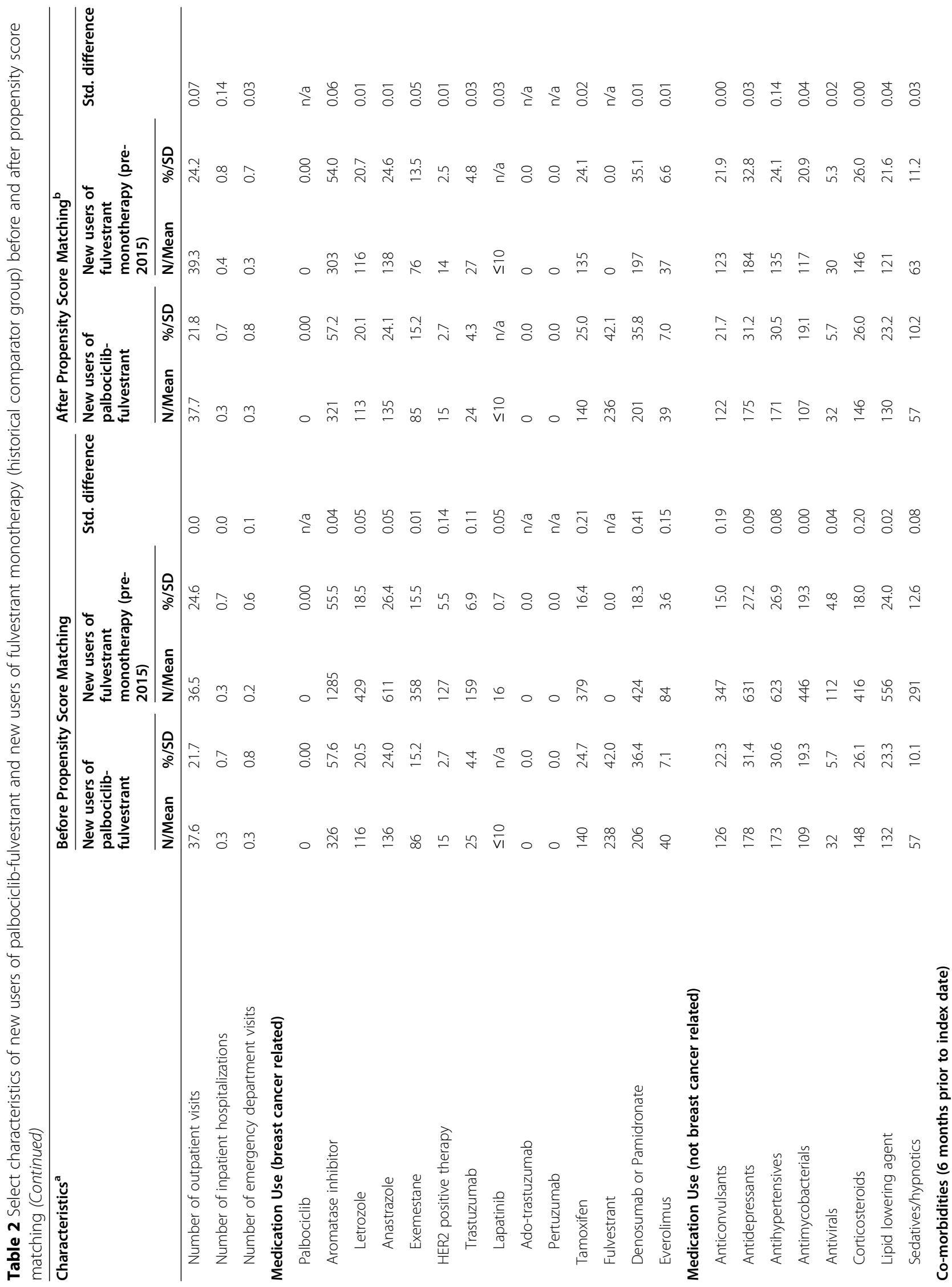




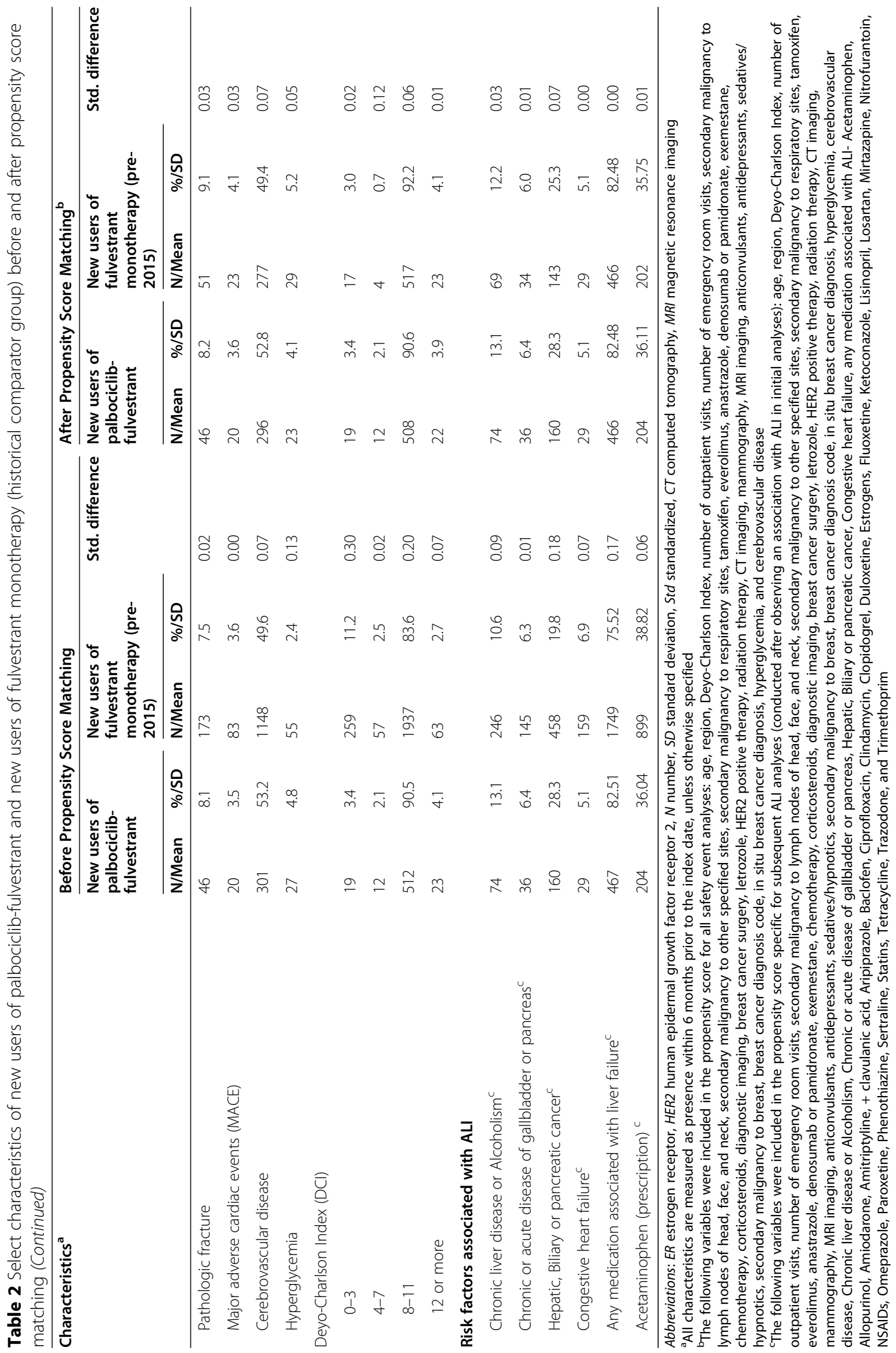


propensity score matching, new users of palbociclibfulvestrant $(n=565$; person-years of follow-up $=322)$ and the historical comparator group of new users of fulvestrant monotherapy $(n=2316$, person-years of followup $=1686$ ) were similar on many factors related to cancer therapy history, such as chemotherapy and radiation therapy, specific types of hormone therapy use, and most co-morbidities (Table 2). Regarding differences between the groups, new fulvestrant users were older than palbociclib-fulvestrant users (mean ages 64 vs. 59), while the palbociclib-fulvestrant users were more likely to have a secondary malignancy (metastases) to respiratory and digestive systems $(45.4 \%$ vs. $33.7 \%)$ and other specified sites $(81.8 \%$ vs. $73.8 \%)$, a higher DCI score ( 8.5 vs. 7.9$)$, and use of certain breast cancer medications, such as tamoxifen, everolimus, denosumab, or pamidronate (Table 2). After propensity score matching, these factors were balanced with standardized differences $<0.10$ (Table 2 and Supplemental Table 4).

When compared to a propensity score-matched historical group of new users of fulvestrant monotherapy $(n=$ 561), new users of palbociclib-fulvestrant $(n=561)$ were more likely to develop neutropenia $(\mathrm{HR}=7.8,95 \% \mathrm{CI}=$ 4.7-13.0), leukopenia ( $\mathrm{HR}=6.4,95 \% \mathrm{CI}=1.9-21.9)$, stomatitis and mucositis $(\mathrm{HR}=5.0,95 \% \mathrm{CI}=1.1-23.1)$, ALI $(\mathrm{HR}=4.8,95 \% \mathrm{CI}=1.4-16.9)$, anemia $(\mathrm{HR}=1.8,95 \%$ $\mathrm{CI}=1.4,-2.3)$, and $\mathrm{QT}$ prolongation $(\mathrm{HR}=1.8,95 \% \mathrm{CI}=$
0.9-3.5) (Table 3). Incidence rates of other safety event rates were similar among new users of palbociclibfulvestrant and new users of fulvestrant monotherapy, including serious infections, type 2 diabetes mellitus, second primary cancers, and pulmonary embolism (Table 3 and Supplemental Table 5).

\section{Additional ALI analyses}

Before propensity score matching, several ALI risk factors were more prevalent in the palbociclib-fulvestrant group than the fulvestrant monotherapy group (e.g., medication associated with ALI: $82.5 \%$ vs. $75.5 \%$; chronic liver disease or alcoholism: $13.1 \%$ vs. $10.6 \%$ ). After propensity score matching, covariates identified as important risk factors for ALI were balanced between the two groups (standardized differences $<0.10$; Table 2 and Supplemental Table 6).

Prior to propensity score matching we observed an elevated risk for the primary ALI endpoint, (unadjusted $\mathrm{HR}=2.8,95 \% \mathrm{CI}=1.4$, 5.6; Fig. 1). After propensity score matching, there were 18 cases of ALI in both groups combined using the primary algorithm, 20 cases using the original algorithm (including elevated aminotransferases), 49 cases using the sensitive algorithm, and 0 cases using the specific algorithm. For the primary ALI endpoint, the incidence was 4.0 per 100 person-years in the palbociclib-fulvestrant group, and 1.2 per 100

Table 3 Select $^{\mathrm{a}}$ Incidence Rates and Propensity Score Adjusted ${ }^{\mathrm{b}}$ Hazard Ratios of the Safety Events Comparing New Users of Palbociclib and Fulvestrant and New Users of Fulvestrant Monotherapy

\begin{tabular}{|c|c|c|c|c|c|c|c|c|c|}
\hline \multirow[t]{4}{*}{ Safety Event ${ }^{c}$} & \multicolumn{9}{|c|}{ After Propensity Score Matching $^{\text {b }}$} \\
\hline & \multirow{2}{*}{\multicolumn{3}{|c|}{$\begin{array}{l}\text { New users of palbociclib-fulvestrant } \\
(n=561) \\
\text { IR (per } 100 \text { person-years) }\end{array}$}} & \multirow{2}{*}{\multicolumn{3}{|c|}{$\begin{array}{l}\text { Historical new users of fulvestrant monotherapy } \\
(n=561)\end{array}$}} & \multicolumn{3}{|c|}{$\begin{array}{l}\text { Hazard Ratio } \\
\text { Estimates }\end{array}$} \\
\hline & & & & & & & \multirow[t]{2}{*}{ HR } & \multirow{2}{*}{$\begin{array}{l}95 \% \\
\text { LCL }\end{array}$} & \multirow{2}{*}{$\begin{array}{l}95 \% \\
\text { UCL }\end{array}$} \\
\hline & $\overline{I R}$ & $95 \% \mathrm{LCL}$ & $95 \%$ UCL & $\overline{I R}$ & $95 \% \mathrm{LCL}$ & $95 \%$ UCL & & & \\
\hline Anemia & 47.7 & 40.0 & 56.5 & 26.2 & 21.1 & 32.2 & 1.8 & 1.4 & 2.3 \\
\hline Neutropenia & 36.7 & 30.0 & 44.5 & 4.6 & 2.7 & 7.4 & 7.8 & 4.7 & 13.0 \\
\hline Serious infection & 25.7 & 20.4 & 32.1 & 22.9 & 18.2 & 28.5 & 1.1 & 0.8 & 1.5 \\
\hline Thrombocytopenia & 10.5 & 7.2 & 14.7 & 4.6 & 2.7 & 7.4 & 2.3 & 1.3 & 4.1 \\
\hline QT prolongation & 6.4 & 3.9 & 9.9 & 3.5 & 1.9 & 6.0 & 1.8 & 0.9 & 3.5 \\
\hline Leukopenia & 5.4 & 3.1 & 8.6 & 0.8 & 0.2 & 2.4 & 6.4 & 1.9 & 21.9 \\
\hline Pulmonary embolism & 4.4 & 2.4 & 7.4 & 4.1 & 2.3 & 6.7 & 1.0 & 0.5 & 2.1 \\
\hline Acute liver injury $(A L I)^{c}$ & 4.1 & 2.2 & 6.9 & 0.8 & 0.2 & 2.4 & 4.8 & 1.4 & 16.9 \\
\hline Stomatitis and mucositis & 2.8 & 1.3 & 5.3 & 0.5 & 0.1 & 1.7 & 5.0 & 1.1 & 23.1 \\
\hline
\end{tabular}

Abbreviations: IR incidence rate, $L C L$ lower confidence limit, $U C L$ upper confidence limit, $H R$ hazard ratio

${ }^{a}$ Results for all safety events are provide in supplemental Table 5 . Safety events selected for this table include notable safety events on the palbociclib product label or those of particular interest

${ }^{\mathrm{b}}$ The following variables were included in the propensity score: age, region, Deyo-Charlson Index (DCI), number of outpatient visits, number of emergency room visits, secondary malignancy (i.e. metastases) to lymph nodes of head, face, and neck, secondary malignancy to other specified sites, secondary malignancy to respiratory and digestive sites (including to the liver), tamoxifen dispensing, everolimus dispensing, anastrazole dispensing, denosumab or pamidronate dispensing, exemestane dispensing, chemotherapy dispensing, corticosteroids dispensing, diagnostic imaging, breast cancer surgery, letrozole dispensing, HER2 positive therapy, radiation therapy, CT (computed tomography) imaging, mammography, MRI (magnetic resonance imaging), anticonvulsants, antidepressants, sedatives/hypnotics, secondary malignancy to breast, breast cancer diagnosis code, in situ breast cancer diagnosis, hyperglycemia, and cerebrovascular disease "All algorithm definitions are provided in Supplemental Table 1. The "original" ALI algorithm for this active surveillance study is noted in this table as the "ALI or elevation of transaminases (ALI definition 2)" 


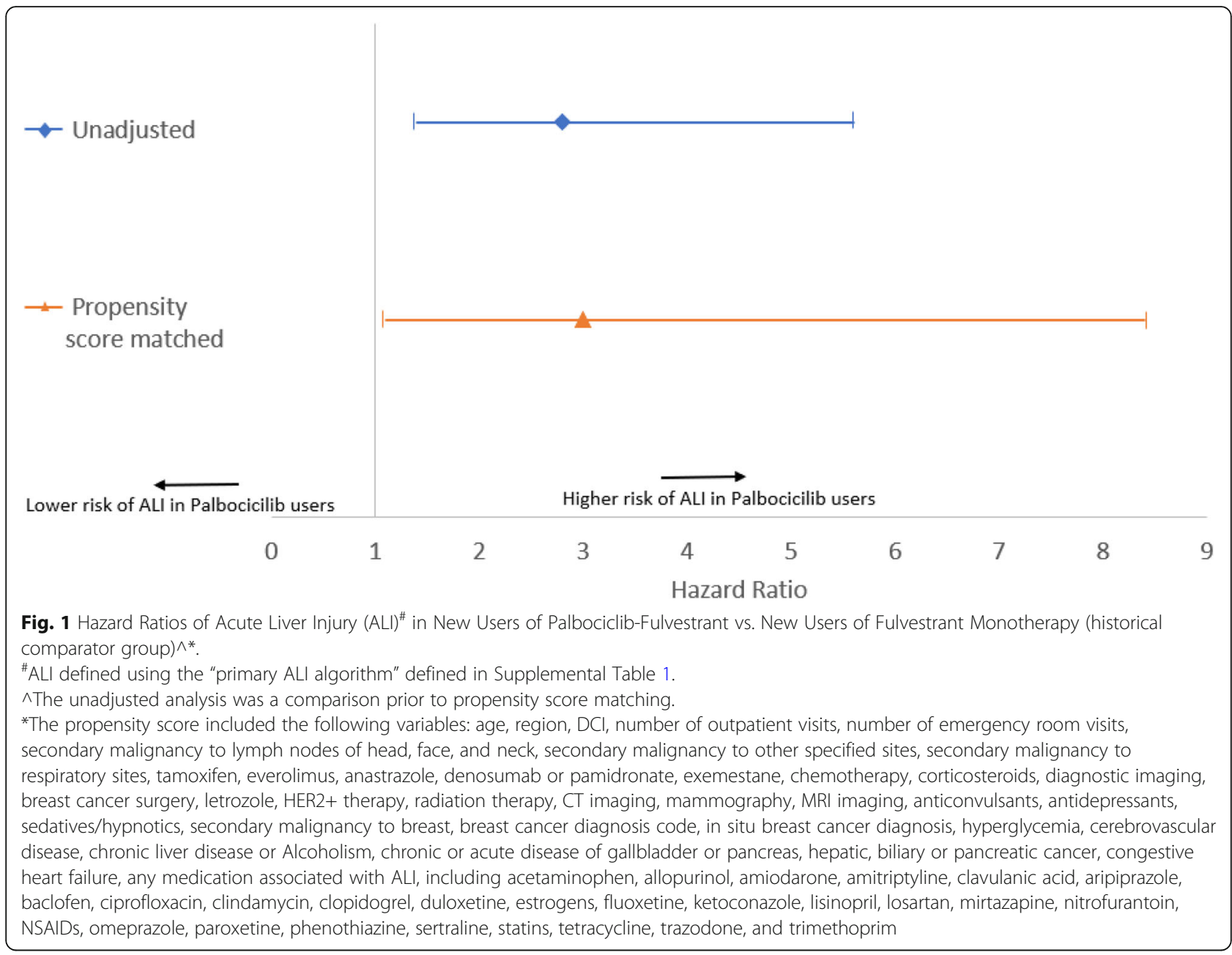

person-years in the fulvestrant monotherapy historical comparator group (unadjusted $\mathrm{HR}=2.8,95 \% \mathrm{CI}=1.4$, 5.6; Fig. 1). Effect estimates were imprecise but similarly elevated for each algorithm (Primary algorithm: $\mathrm{HR}=$ 3.0, $95 \% \mathrm{CI}=1.1-8.4$; original algorithm: $\mathrm{HR}=2.2,95 \%$ $\mathrm{CI}=0.9,5.4$; sensitive algorithm: $\mathrm{HR}=4.6,95 \% \mathrm{CI}=2.3$, 9.1 (Supplemental Table 7, and Fig. 1)).

For the contemporaneous fulvestrant comparator group, we identified 961 new users of fulvestrant monotherapy between 01 February 2015 and 30 September 2017. Before matching, the contemporaneous fulvestrant group and the palbociclib-fulvestrant group were imbalanced with regard to several covariates (e.g., $55 \%$ vs. $31 \%$ greater than age 65 , and $35 \%$ vs. $45 \%$ had metastases to the respiratory and digestive systems; Supplemental Table 8), and we could find matches for only 292 of the palbociclib users.

As a result of the initial imbalance between the palbociclib-fulvestrant users and the contemporaneous comparators, propensity score matching reduced the number of cases available for analysis (e.g. 24 vs. $<10$ cases of ALI in the combined treatment groups using the primary algorithm). Before matching, the incidence of ALI using the primary algorithm was 4.0 per 100 person-years in the palbociclib-fulvestrant group and 2.0 per 100 person-years in the fulvestrant monotherapy contemporaneous comparator group. The unadjusted HR was 2.1, 95\% CI =0.9-4.7. Effect estimates were imprecise after propensity score matching (primary algorithm $\mathrm{HR}=0.5,95 \% \mathrm{CI}=0.1-2.2$ ). Results were similar for the other ALI algorithms (Supplemental Table 9). Additional analyses did not indicate any important temporal trend in incidence of ALI in the HIRD (Supplemental Table 10).

For the ALI validation component of this study, we requested medical records for 138 of the 185 patients meeting any of the ALI algorithms, who did not have health plan-based privacy restrictions; 52 of which were obtained. Among the 29 cases meeting the primary ALI claims algorithm with adjudicated results, 21 were confirmed as ALI cases, the others were confirmed as noncases or remained provisional due to lack of sufficient information in the record. The PPV of the primary ALI algorithm among confirmed cases and non-cases was 
84\% (95\% CI $=64-95 \%$ ), while the other two ALI algorithms had lower PPVs (72 and 73\%; Supplementary Table 11).

\section{Sensitivity analyses}

To evaluate possible unmeasured confounding, we calculated the E-value, which is the minimum strength of the palbociclib-confounder and the confounder-ALI associations needed to explain the observed association between palbociclib-fulvestrant and ALI (i.e. for residual confounding to attenuate the association to the null value, $H R=1.0)$. For the association between palbociclib-fulvestrant and ALI in the historical comparator analysis $(\mathrm{HR}=3.0,95 \% \mathrm{CI}=1.1-8.4)$, a risk factor would need to have an association of at least relative risk $(R R)=5.5$ between palbociclib-fulvestrant and the confounder, and between the confounder and ALI to explain the observed association (Supplemental Figure 1).

We evaluated outcome misclassification by adjusting our comparative estimates by PPVs from the validation component of this study. The results adjusting for PPV were similar to the main results (Supplemental Table 12). In a hypothetical scenario where we assume $100 \%$ sensitivity and 100\% PPV in the fulvestrant monotherapy group, the amount of differential outcome misclassification needed to attenuate the palbociclib-fulvestrant ALI association $(\mathrm{HR}=3.0,95 \% \mathrm{CI}=1.1-8.4)$ to the null value indicating no association would require a PPV of $33 \%$ in palbociclib-fulvestrant group.

\section{Discussion}

This appears to be the first epidemiologic study of palbociclib safety in a real-world setting that compared new users of palbociclib-fulvestrant to a historical comparator of new users receiving fulvestrant monotherapy, and supports findings from clinical trials that myelosuppression events, anemia, thrombocytopenia, and stomatitis and mucositis are more frequent with palbociclibfulvestrant use [1-3, 19-21]. The myelosuppression events, neutropenia and leukopenia, were the most strongly associated with palbociclib-fulvestrant use (HRs $>6$ ). In addition, we found a 2-fold increased risk of QT prolongation and a 3-fold increased risk of ALI with palbociclib-fulvestrant use, while not observing increased rates of pulmonary embolism or serious infections.

In the two phase 3 trials of palbociclib, grade 3/4 ALT elevations occurred in $2 \%$ of the palbociclib group (19/ 789 patients with advanced breast cancer) with no grade $3 / 4$ ALT elevations $(0 / 394)$ in the comparator (endocrine monotherapy) group $(p=0.001)[1]$, despite the fact that baseline liver metastases were somewhat more common in the comparator group [22]. In the PALOMA 3 trial, grade 3 or higher hepatic adverse events included two cases of hepatic failure and one drug-induced liver injury in the palbociclib-fulvestrant arm $[n=345]$, while no such events were included in the comparator arm [ $n=172]$ [19]. The increased risk of ALI reported here is consistent with imbalances in grade 3/4 ALT elevations in clinical trials, and has been hypothesized in recent reports [23-26]. Additionally, ribociclib and abemaciclib, the two other approved CDK4/6 inhibitors, carry hepatotoxicity warnings in their US package inserts, suggesting hepatotoxicity may be common to this class of medication [27, 28]. Both these warnings advise performing liver function tests (LFTs) before drug initiation and during therapy $[27,28]$.

Sensitivity analyses supported a positive association between palbociclib-fulvestrant and ALI with the exception of adjusted analyses using a contemporaneous comparator of fulvestrant monotherapy. Owing to lack of comparability between the palbociclib-fulvestrant group and the contemporaneous comparator, we could find matches for only $292(52 \%)$ palbociclib patients, as compared with 565 (>99\%) palbociclib patients who could be matched to the historical comparators. Further, even after matching, there were still differences between the contemporaneous comparators and the palbociclib users; for example, patients in the contemporaneous comparator group were older than palbociclib-treated patients. The high rates of ALI in the contemporaneous comparator group (twice that of the palbociclib group for the primary algorithm) and imprecise HRs reflect these constraints of small size and noncomparability.

There are several limitations to this study including small sample size, exposure and outcome misclassification, and confounding. Except for ALI, safety outcomes were not validated with medical records, and while propensity scores can control for numerous measured confounders, they do not control for confounding from unmeasured factors unless they are associated with the measured covariates. The secondary malignancy (metastases) codes available indicate only the presence or absence of metastases to broad anatomic sites, but do not provide information on the exact site of metastatic disease, the extent of metastases and/or the metastatic tumor burden. Sensitivity analyses suggested that it would require a particularly strong association with an unmeasured confounder (e.g., extent of metastases, liver involvement, extent of visceral disease, etc.) that is strongly associated with ALI apart from its association with 'measured risk factors (e.g., presence of metastasis), to explain the observed association seen when using the historical fulvestrant monotherapy comparator. Given that ALI is rare in patients with liver metastases [29-31] and given the balance between groups in presence of metastases after propensity score matching, it seems unlikely that there would be a strong residual association 
with the extent of metastases capable of accounting for the observed association.

Finally, while this real-world study is larger than any of the palbociclib clinical trials, the sample size is limited for assessing relatively uncommon events. Imprecision can be seen in the width of the confidence intervals, particularly for rare outcomes such as stomatitis and mucositis, and ALI. Replication in larger studies in other populations (e.g. those with a lower socioeconomic status on Medicaid or older individuals on Medicare) would strengthen these findings, provide further clarity on effect sizes, and would be better able to evaluate any possible interactions palbociclib may have with other medications associated with liver toxicity commonly used in those with advanced stage breast cancer. Additionally, given the severity of ALI [32], if these results are corroborated, further study is warranted on ALI's effect on palbociclib treatment adherence, quality of life, and survival.

\section{Conclusions}

In this real-world study comparing new users of palbociclib-fulvestrant to a historical comparator group of new users of fulvestrant monotherapy, safety events commonly associated with palbociclib use were mostly similar to those identified in randomized trials. An increased risk of ALI is a new finding, although the number of cases was small. This result should be corroborated to better understand the risk in patients treated with palbociclib.

\section{Supplementary Information}

The online version contains supplementary material available at https://doi. org/10.1186/s12885-021-07790-z.

Additional file 1: Supplemental Table 1. Code List for Identifying the Safety Events of Interest in the HIRD*. Supplemental Table 2.

Formation of Study Cohorts. Supplemental Table 3. Incidence of Safety Events in New Users of Palbociclib, Overall and by Subcohort.

Supplemental Table 4. Characteristics of New Users of Palbociclib and Fulvestrant and New Users of Fulvestrant Monotherapy (Historical Comparator Group) Before and After Propensity Score Matching (All evaluated characteristics). Supplemental Table 5. Incidence Rates and Adjusted Hazard Ratios of the Safety Events of Interest in Propensity Score Matched New Users of Palbociclib and Fulvestrant and Historical New Users of Fulvestrant Monotherapy. Supplemental Table 6. Characteristics of New Users of Palbociclib and Fulvestrant and New Users of Fulvestrant Monotherapy (Historical Comparison Group) Before and After Propensity Score Matching (Including ALI Risk Factors).

Supplemental Table 7. Unadjusted and Adjusted Hazard Ratios of ALI in New Users of Palbociclib and Fulvestrant and New Users of Fulvestrant Monotherapy (Historical Comparator). Supplemental Table 8.

Characteristics of New Users of Palbociclib and Fulvestrant and New Users of Fulvestrant Monotherapy (Contemporaneous Comparison Group). Supplemental Table 9. Unadjusted and Adjusted Hazard Ratios of ALI in New Users of Palbociclib and Fulvestrant and New Users of Fulvestrant Monotherapy (Contemporaneous Comparator).

Supplemental Table 10. Incidence of ALI in the HealthCore Integrated Database (HIRD) Between April 2014 and March 2017. Supplemental Table 11. ALI Algorithm Signal Refinement - Validation of Claims
Algorithms Compared to Medical Record Adjudication. Supplemental Table 12. PPV Adjusted Hazard Ratios of ALI in New Users of Palbociclib and Fulvestrant and New Users of Fulvestrant Monotherapy (Historical Comparator). Supplemental Figure 1. E-value to Explain the Association Between Palbociclib-Fulvestrant and the Primary ALI algorithm (in Historical Fulvestrant Analyses)

\section{Abbreviations}

HR: Hormone receptor; HER2: Human epidermal growth factor receptor 2; HIRD: HealthCore Integrated Research Database; ALI: Acute liver injury; HR: Hazard ratio; Cl: Confidence interval; ALT: Alanine aminotransferase; CDK4/6: Cyclin-dependent kinase 4/6; US: United States; FDA: Food Drug Administration; AEs: Adverse events; ICD-9/10: International Classification of Diseases, Ninth and Tenth Revisions; AST: Aspartate amino transferase; ALP: Abnormal alkaline phosphatase; PPV: Positive predictive value; LFTs: Liver function tests; IRB: Institutional Review Board; N: Number; SD: Standard deviation; ER: Estrogen receptor; CT: Computed tomography; Std: Standardized; MRI: Magnetic resonance imaging; IR: Incidence rate; LCL: Lower confidence limit; UCL: Upper confidence limit

\section{Acknowledgments}

Mark Paulin, Rebecca Conto, Caroline Flessa, and Karnaiditya Rana are employees of HealthCore who provided operational support on the medical record validation component of the study. HealthCore received funding from Pfizer for the conduct of this study.

\section{Authors' contributions}

Study conception: DCB, CD, SL; Study design: DCB, CD, SL; Analysis: AJA, RY, $\mathrm{DCB}$; Interpretation of data: DCB, CD, SL, DT, RY, AJA, AS, JWL, JWF; Draft or revision of manuscript: DCB, CD, SL, DT, RY, AJA, AS, JWL, JWF. All authors have read and approved the manuscript.

\section{Funding}

This study was funded by Pfizer Inc. One of the co-authors (CD) is an employee of Pfizer Inc. - and thus the company reviewed and commented on material related to study conception and design. They were also involved in the interpretation of the data, and in the revision of this manuscript.

\section{Availability of data and materials}

The datasets generated during and/or analysed during the current study are not publicly available due privacy regulations.

Ethics approval and consent to participate

All procedures performed in studies involving human participants were in accordance with the ethical standards of the institutional and/or national research committee and with the 1964 Helsinki declaration and its later amendments or comparable ethical standards.This study was approved by the New England Institutional Review Board (IRB Study No. 3325). The current study was designed as an analysis based on claims data from a large insured population in the US. There was no active enrollment or active follow-up of study subjects, and no data were collected directly from individuals. The HIPAA Privacy Rule permits PHI in a limited data set to be used or disclosed for research, without individual authorization, if certain criteria are met (further described 45 CFR Part 160 and Subparts A and E of Part 164). Thus informed consent was not required.

\section{Consent for publication}

Not applicable.

\section{Competing interests}

DCB, AJA, DHT, and SL are employees of HealthCore, Inc., and were paid consultants to Pfizer in connection with the development of this manuscript. RY was an employee of HealthCore, Inc. at the time of this study, and is now an employee of Anthem Inc. CD is an employee of Pfizer Inc. AS and JHL were paid consultants to HealthCore, Inc. for the assessment of ALI events but received no compensation for authorship. JWF is an independent consultant in drug-induced liver injury for several pharmaceutical companies, none which have drugs in development or on the market in the same class or the same indication. 


\section{Author details}

'HealthCore, Inc., 123 Justison Street, Suite 200, Wilmington, DE 19801, USA. ${ }^{2}$ Pfizer Inc., New York, NY, USA. ${ }^{3}$ Anthem, Inc., Andover, MA, USA. ${ }^{4}$ Duke University School of Medicine, Durham, NC, USA. ${ }^{5}$ Georgetown University School of Medicine, Washington, DC, USA. ${ }^{6}$ University of Connecticut Health Center, Farmington, CT, USA.

Received: 24 September 2020 Accepted: 5 January 2021

Published online: 25 January 2021

\section{References}

1. IBRANCE (palbociclib) [package insert]. New York: Pfizer Inc.; 2017. Available from: https://www.accessdata.fda.gov/drugsatfda_docs/label/2017/207103 s004lbl.pdf. Accessed 15 Sept 2020

2. Turner NC, Ro J, Andre F, Loi S, Verma S, Iwata H, et al. Palbociclib in hormone-receptor-positive advanced breast Cancer. N Engl J Med. 2015; 373(3):209-19.

3. Finn RS, Martin M, Rugo HS, Jones S, Im SA, Gelmon K, et al. Palbociclib and Letrozole in advanced breast Cancer. N Engl J Med. 2016;375(20):1925-36.

4. FEMERA (letrozole) [package insert]. Basel: Novartis; 1997. Available from: https://www.accessdata.fda.gov/drugsatfda_docs/label/2014/020726s027lbl. pdf. Accessed 15 Sept 2020.

5. Lo Re V 3rd, Haynes K, Goldberg D, Forde KA, Carbonari DM, Leidl KB, et al. Validity of diagnostic codes to identify cases of severe acute liver injury in the US Food and Drug Administration's mini-sentinel distributed database. Pharmacoepidemiol Drug Saf. 2013;22(8):861-72.

6. Lo Re V, Carbonari DM, Saine ME, Newcomb CW, Roy JA, Liu Q, et al. Postauthorization safety study of the DPP-4 inhibitor saxagliptin: a largescale multinational family of cohort studies of five outcomes. BMJ Open Diabetes Res Care. 2017;5(1):e000400.

7. Chan KA, Truman A, Gurwitz JH, Hurley JS, Martinson B, Platt R, et al. A cohort study of the incidence of serious acute liver injury in diabetic patients treated with hypoglycemic agents. Arch Intern Med. 2003;163(6): $728-34$

8. FDA Working Group. PhRMA/FDA/AASLD drug-induced hepatotoxicity white paper: postmarketing considerations. Silver Spring: US Department of Health and Human Services, Food and Drug Administration; 2000.

9. Navarro VJ, Senior JR. Drug-related hepatotoxicity. N Engl J Med. 2006; 354(7):731-9.

10. Panozzo CA, Woodworth TS, Welch EC, Huang TY, Her QL, Haynes K, et al. Early impact of the ICD-10-CM transition on selected health outcomes in 13 electronic health care databases in the United States. Pharmacoepidemiol Drug Saf. 2018;27(8):839-47.

11. Austin PC. Balance diagnostics for comparing the distribution of baseline covariates between treatment groups in propensity-score matched samples. Stat Med. 2009;28(25):3083-107.

12. Schoenberg BS. Calculating confidence intervals for rates and ratios. Neuroepidemiology. 1983;2:257-65.

13. Brookhart MA, Schneeweiss S, Rothman KJ, Glynn RJ, Avorn J, Sturmer T. Variable selection for propensity score models. Am J Epidemiol. 2006: 163(12):1149-56

14. Rosenbaum PRR DB. The cental role of the propensity score in observational studies for causal effects. Biometrika. 1983;70(1):41-55.

15. Austin PC. An introduction to propensity score methods for reducing the effects of confounding in observational studies. Multivar Behav Res. 2011; 46(3):399-424.

16. VanderWeele TJ, Ding P. Sensitivity analysis in observational research: introducing the E-value. Ann Intern Med. 2017;167(4):268-74.

17. Brenner $\mathrm{H}$, Gefeller $\mathrm{O}$. Use of the positive predictive value to correct for disease misclassification in epidemiologic studies. Am J Epidemiol. 1993; 138(11):1007-15.

18. Beachler DC, de Luise C, Yin R, Gangemi K, Cochetti PT, Lanes S. Predictive model algorithms identifying early and advanced stage ER+/HER2- breast cancer in claims data. Pharmacoepidemiol Drug Saf. 2019;28(2):171-8.

19. Cristofanilli M, Turner NC, Bondarenko I, Ro J, Im SA, Masuda N, et al. Fulvestrant plus palbociclib versus fulvestrant plus placebo for treatment of hormone-receptor-positive, HER2-negative metastatic breast cancer that progressed on previous endocrine therapy (PALOMA-3): final analysis of the multicentre, double-blind, phase 3 randomised controlled trial. Lancet Oncol. 2016;17(4):425-39.
20. Finn RSR HS, Gelmon K, Cristofanilli M, Colleoni M, Loi S, Schnell P, Lu DR, Theall KP, Mori A, Gauthier E, Bananis E, Turner NC, Dieras V. Abstract P1-1916: Long-term pooled safety analysis of palbociclib in combination with endocrine therapy for hormone receptor-positive/human epidermal growth factor receptor 2-negative advanced breast cancer: An updated analysis. Cancer Res. 2020;80:P1-19-6.

21. Dieras V, Rugo HS, Schnell P, Gelmon K, Cristofanilli M, Loi S, et al. Longterm pooled safety analysis of Palbociclib in combination with endocrine therapy for HR+/HER2- advanced breast Cancer. J Natl Cancer Inst. 2019; $111(4): 419-30$

22. Turner NC, Finn RS, Martin M, Im SA, DeMichele A, Ettl J, et al. Clinical considerations of the role of palbociclib in the management of advanced breast cancer patients with and without visceral metastases. Ann Oncol. 2018;29(3):669-80

23. Vuppalanchi R, Saxena R, Storniolo AMV, Chalasani N. Pseudocirrhosis and liver failure in patients with metastatic breast cancer after treatment with palbociclib. Hepatology. 2017;65(5):1762-4.

24. Roberts BAI M, Stone E. Elevated liver function tests in a patient on palbociclib and fulvestrant. J Commun Supp Oncol. 2018;16(6):e277-9.

25. Raschi E, De Ponti F. Strategies for early prediction and timely recognition of drug-induced liver injury: the case of Cyclin-dependent kinase 4/6 inhibitors. Front Pharmacol. 2019:10:1235.

26. Atallah R, Parker NA, Hamouche K, Truong QV, Dingwall M. Palbociclibinduced liver failure. Kans J Med. 2020;13:81-2.

27. FDA. Kisqali (ribociclib) 2017. Available from: https://www.accessdata.fda. gov/drugsatfda_docs/label/2017/209092s000lbl.pdf.

28. VERZENIO ${ }^{T M}$ (abemaciclib) package insert Indianapolis, IN. Available from: https:/www.accessdata.fda.gov/drugsatfda_docs/label/2017/208716s000lbl. pdf. Accessed 15 Sept 2020.

29. Rowbotham $D$, Wendon J, Williams R. Acute liver failure secondary to hepatic infiltration: a single Centre experience of 18 cases. Gut. 1998;42(4): $576-80$

30. Hanamornroongruang S, Sangchay N. Acute liver failure associated with diffuse liver infiltration by metastatic breast carcinoma: a case report. Oncol Lett. 2013;5(4):1250-2.

31. Nazario HE, Lepe R, Trotter JF. Metastatic breast cancer presenting as acute liver failure. Gastroenterol Hepatol (N Y). 2011;7(1):65-6.

32. Andrade RJ, Chalasani N, Bjornsson ES, Suzuki A, Kullak-Ublick GA, Watkins PB, et al. Drug-induced liver injury. Nat Rev Dis Primers. 2019:5(1):58.

\section{Publisher's Note}

Springer Nature remains neutral with regard to jurisdictional claims in published maps and institutional affiliations.

Ready to submit your research? Choose BMC and benefit from:

- fast, convenient online submission

- thorough peer review by experienced researchers in your field

- rapid publication on acceptance

- support for research data, including large and complex data types

- gold Open Access which fosters wider collaboration and increased citations

- maximum visibility for your research: over $100 \mathrm{M}$ website views per year

At BMC, research is always in progress.

Learn more biomedcentral.com/submissions 